\title{
DUST ATTENUATION IN UV-SELECTED STARBURSTS AT HIGH REDSHIFT AND THEIR LOCAL COUNTERPARTS: IMPLICATIONS FOR THE COSMIC STAR FORMATION RATE DENSITY
}

\author{
Roderik A. Overzier ${ }^{1}$, Timothy M. Heckman ${ }^{2}$ Jing Wang $^{1}$, Lee Armus ${ }^{3}$, Veronique Buat $^{4}$, Justin Howell $^{3}$, \\ Gerhardt Meurer $^{5}$, Mark Seibert ${ }^{6}$, Brian Siana ${ }^{7}$, Antara Basu-Zych ${ }^{8}$, Stéphane Charlot $^{9}$, Thiago S. Gonçalves ${ }^{7}$, \\ D. Christopher Martin ${ }^{7}$, James D. Neill ${ }^{7}$, R. Michael Rich ${ }^{10}$, SAmir Salim $^{11}$, and David Schiminovich ${ }^{12}$ \\ ${ }^{1}$ Max-Planck-Institut for Astrophysics, D-85748 Garching, Germany; overzier@ mpa-garching.mpg.de \\ ${ }^{2}$ Department of Physics and Astronomy, The Johns Hopkins University, 3400 North Charles Street, Baltimore, MD 21218, USA \\ ${ }^{3}$ Spitzer Science Center, Caltech, MS 220-6, Pasadena, CA 91125, USA \\ ${ }^{4}$ Laboratoire d'Astrophysique de Marseille, OAMP, Université Aix-marseille, CNRS, 38 rue Frédéric Joliot-Curie, 13388 Marseille Cedex 13, France \\ ${ }^{5}$ ICRAR/University of Western Australia, 35 Stirling Highway, Crawley, WA 6009, Australia \\ ${ }^{6}$ Observatories of the Carnegie Institution of Washington, 813 Santa Barbara Street, Pasadena, CA 91101, USA \\ ${ }^{7}$ California Institute of Technology, MS 249-17, Pasadena, CA 91125, USA \\ ${ }^{8}$ NASA Goddard Space Flight Center, Laboratory for X-ray Astrophysics, Greenbelt, MD 20771, USA \\ ${ }^{9}$ PMC Univ Paris 06, UMR7095, Institut d'Astrophysique de Paris, F-75014 Paris, France \\ ${ }^{10}$ Department of Physics and Astronomy, Division of Astronomy and Astrophysics, University of California, Los Angeles, CA 90095-1562, USA \\ ${ }^{11}$ National Optical Astronomical Observatories, 950 N. Cherry Avenue, Tucson, AZ 85719, USA \\ ${ }^{12}$ Department of Astronomy, Columbia University, MC 2457, 550 West 120th Street, New York, NY 10027, USA \\ Received 2010 November 4; accepted 2010 November 24; published 2010 December 10
}

\begin{abstract}
We present a new analysis of the dust obscuration in starburst galaxies at low and high redshifts. This study is motivated by our unique sample of the most extreme UV-selected starburst galaxies in the nearby universe $(z<0.3)$, found to be good analogs of high-redshift Lyman break galaxies (LBGs) in most of their physical properties. We find that the dust properties of the Lyman break analogs (LBAs) are consistent with the relation derived previously by Meurer et al. (M99) that is commonly used to dust-correct star formation rate (SFR) measurements at a very wide range of redshifts. We directly compare our results with high-redshift samples (LBGs, " $B z K$," and submillimeter galaxies at $z \sim 2-3$ ) having IR data either from Spitzer or Herschel. The attenuation in typical LBGs at $z \sim 2-3$ and LBAs is very similar. Because LBAs are much better analogs to LBGs compared to previous local star-forming samples, including M99, the practice of dust-correcting the SFRs of high-redshift galaxies based on the local calibration is now placed on a much more solid ground. We illustrate the importance of this result by showing how the locally calibrated relation between UV measurements and extinction is used to estimate the integrated, dust-corrected SFR density at $z \simeq 2-6$.
\end{abstract}

Key words: dust, extinction - galaxies: high-redshift - galaxies: peculiar - galaxies: starburst

Online-only material: color figures

\section{INTRODUCTION}

The ultraviolet (UV) and far-infrared (far-IR) emission of star-forming galaxies (SFGs) offers one of the most direct estimates of their star formation rate (SFR). The fraction of light emerging in the IR relative to that in the UV is modulated primarily by the amount of dust seen by young stars (Meurer et al. 1999; Calzetti et al. 2000; Buat et al. 2002; Burgarella et al. 2005; Seibert et al. 2005; Treyer et al. 2007), with secondary effects due to, e.g., star formation history and geometry (Charlot \& Fall 2000; Kong et al. 2004; Inoue et al. 2006; Boissier et al. 2007; Johnson et al. 2007; Salim et al. 2007; Panuzzo et al. 2007; Cortese et al. 2008; Boselli et al. 2010). For a relatively young galaxy forming stars at a stable rate, the SFR is proportional to the UV luminosity (Leitherer \& Heckman 1995), while the degree of reddening of the UV continuum is related to the amount of dust. This reddening can be expressed in terms of the UV slope, $\beta$ with $f_{\lambda} \propto \lambda^{\beta}$, and the total attenuation is given by the ratio IRX $\equiv L_{\mathrm{IR}} / L_{\mathrm{UV}}$ (Meurer et al. 1999, M99). The "IRX $-\beta$ " relation of M99 is based on a large sample of local starburst galaxies and has proven to be an extremely useful tool for estimating total SFRs from UV-only data. For the relation to work, it is implied that the dust must be near the UV sources and has some kind of shell- or screen-like geometry (Gordon et al. 2000).
The IRX relation is particularly important at high redshift. SFR estimates based on IR, X-ray, or radio data are typically available only for the brightest objects (Pope et al. 2006; Siana et al. 2008, 2009; Reddy et al. 2006, 2010, R10), or statistically through stacks (Seibert et al. 2002; Carilli et al. 2008; Reddy et al. 2010, R10; Magdis et al. 2010a, 2010b; Ho et al. 2010; Kurczynski et al. 2010; Rigopoulou et al. 2010). Surveys in the rest-frame UV can simultaneously give $L_{\mathrm{FUV}}$ and $\beta$ and hence the dust-corrected SFR based on the local IRX relation. Using this technique, Bouwens et al. (2009, B09) estimated the $z \sim 3-6$ SFR density from large samples of Lyman break galaxies (LBGs). They found that most of the energy output at $z=2.5-4(z=4-6)$ occurs in the IR (UV), and that the SFR density can be largely recovered by dust-correcting the UV measurements thus demonstrating the importance of UV surveys. However, because local calibrations of the IRX relation are based on galaxies that are very different from typical UVselected starburst galaxies at $z \gtrsim 3$, it is not clear whether they are valid at high redshift. In this letter, we re-investigate the IRX $-\beta$ relation for the first time using a unique population of nearby $(z<0.3)$ starbursts that has been shown to be similar to LBGs in most of their basic physical properties. These "Lyman break analogs" (LBAs) are similar in mass, age, size, metallicity, optical extinction, and SFR (Heckman et al. 2005; Hoopes et al. 2007; Basu-Zych et al. 2007), have similar compact and clumpy 
morphologies (Overzier et al. 2008, 2010), similar kinematics (Basu-Zych et al. 2009; Gonçalves et al. 2010), and a similar feedback-dominated interstellar medium (Overzier et al. 2009, O09). The structure of this letter is as follows. We first present our data and measurements (Section 2). We compare LBAs with low- and high-redshift SFGs having good IR data from Spitzer or Herschel (Section 3), and we discuss the possible implications of our results on the SFR density at high redshift (Section 4).

\section{DATA AND METHODS}

\subsection{Lyman Break Analogs UV and IR Data}

The sample of 31 LBAs from O09 forms the basis of this Letter. FUV luminosities, $L_{\mathrm{FUV}} \equiv \lambda L_{\lambda}$ with $\lambda=1600 \AA$, were calculated from our F150LP $\left(\lambda_{c} \approx 1614 \AA\right)$ data taken with the Hubble Space Telescope (HST) Advanced Camera for Surveys (see Overzier et al. 2008 and O09, for details). The slope of the UV continuum, $\beta_{\mathrm{GALEX}}$, was calculated ${ }^{13}$ from Galaxy Evolution Explorer (GALEX) General Release 6 data in the $\operatorname{FUV}\left(\lambda_{c} \approx 1530 \AA\right)$ and $\operatorname{NUV}\left(\lambda_{c} \approx 2315 \AA\right)$ having total exposure times of $\sim 100-13,000 \mathrm{~s}$. All LBAs are unresolved in the GALEX images, and we measured total magnitudes in a $9^{\prime \prime}$ radius aperture. We perform a small $K$-correction to obtain $L_{\mathrm{FUV}}$ and $\beta_{\text {GALEX }}$ at $z=0$ using a set of starburst templates. In this Letter, we work exclusively with the photometric measure of $\beta$ (as opposed to the "true" $\beta$ obtained from a power-law fit to a UV spectrum), that is most appropriate for comparing with samples for which spectra are usually not available in large numbers.

We use IR data obtained with the Infrared Array Camera (IRAC), the Multi-band Imaging Photometer (MIPS), and the InfraRed Spectrograph (IRS) on the Spitzer Space Telescope (O09; L. Armus et al. 2011, in preparation). At $24 \mu \mathrm{m}$ and shorter wavelengths, point-source photometry was performed on the post-basic calibration data ("pBCD"). At 70 and 160 $\mu \mathrm{m}$, flux densities were obtained by aperture photometry on the filtered $\mathrm{pBCD}$ images. Upper limits were determined from the standard deviation images. The IRS spectra covering observed wavelengths in the range 5-35 $\mu \mathrm{m}$ were normalized to the MIPS $24 \mu \mathrm{m}$ data. We estimate IR luminosities, $L_{\mathrm{IR}}(3-1000 \mu \mathrm{m})$, by fitting our data with the model library of Siebenmorgen \& Krügel (2007, SK07). This method allows us to fit models covering a wide range of physical parameters, thus obtaining a good sense of the range of models allowed by our data within the measurement errors. We simulate our IRAC+MIPS+IRS data set (including measurement errors and upper limits) and calculate $L_{\mathrm{IR}}$ and its error by taking the median and standard deviation of all the templates that best fit the data in the Monte Carlo simulation ${ }^{14}$. Four objects lacking a sufficient number of IR data points were removed from the sample, as well as one object with an (obscured) active nucleus identified in recent optical spectroscopy. The UV and IR measurements for the 26 remaining objects are given in Table 1.

\subsection{Redetermination of the Meurer et al. (1999) Relation}

In order to be able to compare the results from different samples at low and high redshifts in a consistent manner,

$13 \beta_{\mathrm{GALEX}}=\frac{0.4\left(m_{\mathrm{FUV}}-m_{\mathrm{NUV}}\right)}{\log _{10}\left(\lambda_{\mathrm{NUV}} / \lambda_{\mathrm{FUV}}\right)}-2.0$.

14 While our results obtained using SK07 are consistent with those obtained from blackbody fitting, we note that the SK07 library generally performs better for LBAs compared to the more commonly used empirical library from Chary \& Elbaz (2001). The latter often failed to simultaneously fit the mid-IR and the far-IR dust emission, presumably due to the fact that the LBAs show a greater spectral variation than typical IR-selected starbursts in the local universe.

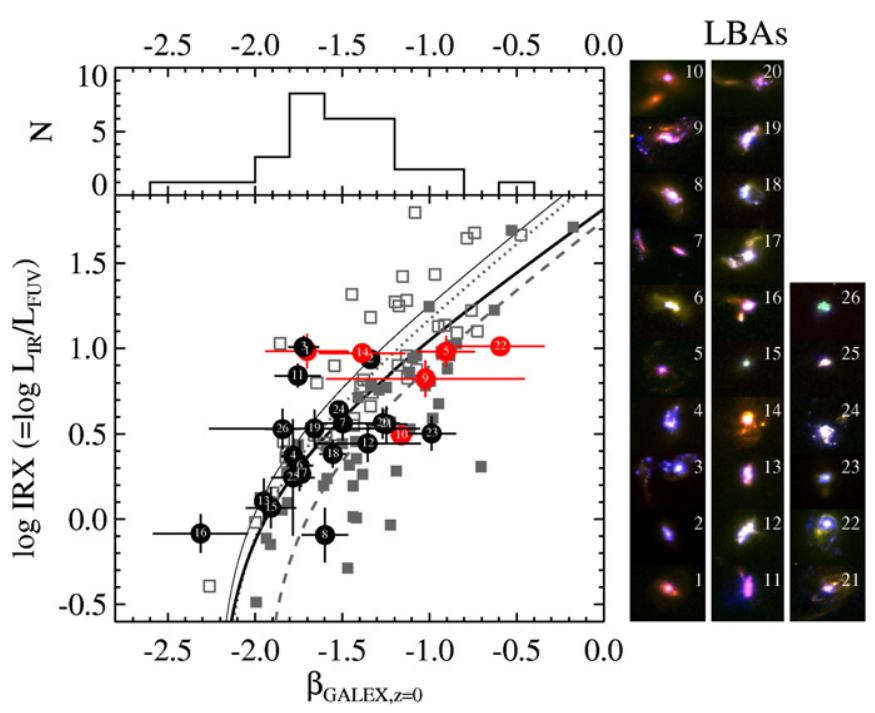

Figure 1. Main panel: the relation between the UV slope and "IRX" for LBAs (large symbols). LBAs referred to as "dominant compact objects" (DCOs; O09) are marked in red, and HST morphologies from O09 are shown on the right. Open and filled squares indicate M99 galaxies re-measured using GALEX data (Section 2.2) using a small aperture similar to the original IUE measurements (open squares), and using an aperture enclosing the entire galaxy (filled squares). Fits to the data are indicated (thick black line: IRX $\mathrm{X}_{\mathrm{LBA}}$; gray dotted line: IRX $\mathrm{M}_{\mathrm{M} \text {,inner }}$; gray dashed line: $\mathrm{IRX}_{\mathrm{M} 99 \text {,total }}$. The original relation from M99, $\mathrm{IRX}_{\mathrm{M} 99,0}$, is shown for comparison (thin black line). Top panel: distribution in $\beta_{z=0}$ for LBAs.

(A color version of this figure is available in the online journal.)

we have re-measured the original M99 IRX $-\beta$ relation using GALEX data and an updated estimate of $L_{\mathrm{IR}}$. Forty-seven galaxies of the M99 sample are covered by GALEX. Details on the photometry will be provided in a forthcoming paper. In brief, we follow the procedures in Wang et al. (2010) carefully masking neighbors and image artifacts, perform background subtraction, and photometry on point-spread function matched images using elliptical apertures out to a maximum radius, $r_{\max }$, of 2.5-5 $\times r_{\text {kron }}$. Because $r_{\max }$ is in most cases much larger than the International UV Explorer (IUE) aperture of $10^{\prime \prime} \times 20^{\prime \prime}$ used by M99, we also measure the inner UV colors and fluxes within a maximum radius of $10^{\prime \prime}$ for comparison with earlier work. We compute $L_{\mathrm{IR}}$ using the Infrared Astronomical Satellite (IRAS) fluxes at 12, 25, 60, and $100 \mu \mathrm{m}$ (Sanders \& Mirabel 1996).

\section{RESULTS}

\subsection{The IRX- $\beta$ Relation of LBAs Versus M99}

In Figure 1 we show the IRX- $\beta$ diagram for LBAs. Panels show the corresponding HST morphologies from O09 and the distribution of UV slopes (top panel). We also show the values measured in Section 2.2 for the M99 galaxies using an aperture radius of $10^{\prime \prime}$ most similar to M99, and within $R_{\max }$ enclosing the entire source. We follow M99 and write IRX in terms of $A_{\text {FUV }}$, the attenuation at $1600 \AA$, and the bolometric corrections to the total light emitted by stars $\left(\mathrm{BC}_{\mathrm{FUV}, *}\right)$ and dust $\left(\mathrm{BC}_{\mathrm{dust}}\right)$ :

$$
\log _{10}(\mathrm{IRX})=\log _{10}\left(10^{0.4 A_{\mathrm{FUV}}}-1\right)+\log _{10} \frac{\mathrm{BC}_{\mathrm{FUV}, *}}{\mathrm{BC}_{\mathrm{dust}}},
$$

with $\mathrm{BC}_{\mathrm{FUV}, *} \approx 1.68$ (M99; Seibert et al. 2005), and $\mathrm{BC}_{\mathrm{dust}} \approx 1$ for our estimate of $L_{\mathrm{IR}}$. We then derive the best-fit relation by performing a linear fit of the form $A_{\mathrm{FUV}}=C_{0}+C_{1} \beta$ to the data in Figure 1. Similar to M99 we exclude galaxies having radii 
Table 1

Lyman Break Analogs: UV and IR Measurements

\begin{tabular}{|c|c|c|c|c|c|c|c|c|c|c|}
\hline $\mathrm{ID}^{\mathrm{a}}$ & $\begin{array}{c}\text { Name } \\
(\text { SDSSJ...) }\end{array}$ & $z$ & $\begin{array}{l}m_{\mathrm{FUV}} \\
(\mathrm{mag})\end{array}$ & $\begin{array}{l}m_{\mathrm{NUV}} \\
(\mathrm{mag})\end{array}$ & $\beta_{\mathrm{obs}}$ & $\beta_{z=0}$ & $\begin{array}{c}\log L_{\mathrm{FUV}} \\
\left(L_{\odot}\right)\end{array}$ & $\begin{array}{c}\log L_{\mathrm{FIR}}{ }^{\mathrm{b}} \\
\left(L_{\odot}\right)\end{array}$ & $\begin{array}{c}\log L_{\mathrm{IR}}{ }^{\mathrm{c}} \\
\left(L_{\odot}\right)\end{array}$ & $\log$ IRX \\
\hline $1^{\dagger}$ & $005439.79+155446.9$ & 0.236 & 20.47 & 20.23 & $-1.44 \pm 0.24$ & -1.69 & $10.24 \pm 0.02$ & $11.03 \pm 0.10$ & $11.22 \pm 0.10$ & $0.98 \pm 0.10$ \\
\hline 2 & $005527.46-002148.7$ & 0.167 & 19.08 & 18.71 & $-1.15 \pm 0.05$ & -1.33 & $10.46 \pm 0.01$ & $11.04 \pm 0.08$ & $11.40 \pm 0.05$ & $0.93 \pm 0.05$ \\
\hline 3 & $015028.40+130858.3$ & 0.147 & 18.48 & 18.33 & $-1.64 \pm 0.09$ & -1.71 & $10.64 \pm 0.01$ & $11.40 \pm 0.08$ & $11.64 \pm 0.06$ & $1.01 \pm 0.07$ \\
\hline 4 & 020356.91-080758.5 & 0.189 & 19.15 & 19.00 & $-1.65 \pm 0.07$ & -1.77 & $10.56 \pm 0.01$ & $10.68 \pm 0.10$ & $10.93 \pm 0.10$ & $0.37 \pm 0.10$ \\
\hline $5^{\dagger}$ & $021348.53+125951.4$ & 0.219 & 19.60 & 18.91 & $-0.46 \pm 0.16$ & -0.89 & $10.65 \pm 0.02$ & $11.38 \pm 0.08$ & $11.63 \pm 0.09$ & $0.98 \pm 0.09$ \\
\hline 6 & $032845.99+011150.8$ & 0.142 & 19.19 & 19.05 & $-1.68 \pm 0.08$ & -1.73 & $10.23 \pm 0.01$ & $10.31 \pm 0.17$ & $10.55 \pm 0.15$ & $0.31 \pm 0.15$ \\
\hline 7 & 035733.99-053719.7 & 0.204 & 19.65 & 19.33 & $-1.27 \pm 0.21$ & -1.49 & $10.47 \pm 0.02$ & $10.81 \pm 0.10$ & $11.03 \pm 0.10$ & $0.56 \pm 0.10$ \\
\hline 8 & 040208.86-050642.0 & 0.139 & 18.89 & 18.68 & $-1.51 \pm 0.13$ & -1.59 & $10.34 \pm 0.02$ & $10.03 \pm 0.20$ & $10.25 \pm 0.16$ & $-0.09 \pm 0.16$ \\
\hline $9^{\dagger}$ & $080232.34+391552.6$ & 0.267 & 20.02 & 19.34 & $-0.45 \pm 0.57$ & -1.01 & $10.68 \pm 0.06$ & $11.23 \pm 0.11$ & $11.50 \pm 0.09$ & $0.82 \pm 0.11$ \\
\hline $10^{\dagger}$ & $080844.26+394852.3$ & 0.091 & 17.98 & 17.59 & $-1.10 \pm 0.06$ & -1.15 & $10.35 \pm 0.01$ & $10.47 \pm 0.07$ & $10.84 \pm 0.03$ & $0.49 \pm 0.03$ \\
\hline 11 & $082001.72+505039.1$ & 0.217 & 19.85 & 19.65 & $-1.55 \pm 0.13$ & -1.74 & $10.47 \pm 0.01$ & $10.98 \pm 0.10$ & $11.31 \pm 0.07$ & $0.84 \pm 0.07$ \\
\hline 12 & $082550.95+411710.2$ & 0.156 & 19.58 & 19.23 & $-1.19 \pm 0.31$ & -1.34 & $10.29 \pm 0.02$ & $10.53 \pm 0.09$ & $10.74 \pm 0.11$ & $0.45 \pm 0.11$ \\
\hline 13 & $083803.72+445900.2$ & 0.143 & & & $-1.92 \pm 0.04$ & -1.94 & $10.44 \pm 0.00$ & $10.32 \pm 0.11$ & $10.54 \pm 0.13$ & $0.10 \pm 0.13$ \\
\hline 15 & $092336.45+544839.2$ & 0.222 & 19.72 & 19.61 & $-1.75 \pm 0.14$ & -1.90 & $10.53 \pm 0.01$ & $10.27 \pm 0.18$ & $10.60 \pm 0.12$ & $0.07 \pm 0.12$ \\
\hline 16 & $092600.40+442736.1$ & 0.181 & 18.69 & 18.83 & $-2.32 \pm 0.27$ & -2.30 & $10.65 \pm 0.02$ & $10.19 \pm 0.17$ & $10.57 \pm 0.11$ & $-0.08 \pm 0.11$ \\
\hline 17 & $093813.49+542825.0$ & 0.102 & 17.83 & 17.72 & $-1.73 \pm 0.05$ & -1.72 & $10.59 \pm 0.00$ & $10.51 \pm 0.09$ & $10.85 \pm 0.08$ & $0.27 \pm 0.08$ \\
\hline 18 & $102613.97+484458.9$ & 0.160 & 19.20 & 18.95 & $-1.43 \pm 0.08$ & -1.54 & $10.35 \pm 0.01$ & $10.50 \pm 0.08$ & $10.74 \pm 0.08$ & $0.38 \pm 0.08$ \\
\hline 19 & $124819.74+662142.6$ & 0.260 & 19.98 & 19.70 & $-1.35 \pm 0.60$ & -1.65 & $10.55 \pm 0.06$ & $10.81 \pm 0.11$ & $11.08 \pm 0.10$ & $0.53 \pm 0.11$ \\
\hline 20 & $135355.90+664800.5$ & 0.198 & 18.99 & 18.54 & $-0.98 \pm 0.10$ & -1.26 & $10.67 \pm 0.02$ & $11.05 \pm 0.08$ & $11.23 \pm 0.09$ & $0.56 \pm 0.09$ \\
\hline 21 & $143417.15+020742.3$ & 0.180 & 19.50 & 19.07 & $-1.01 \pm 0.12$ & -1.24 & $10.44 \pm 0.01$ & $10.72 \pm 0.11$ & $11.00 \pm 0.11$ & $0.56 \pm 0.11$ \\
\hline $22^{\dagger}$ & 210358.74-072802.4 & 0.137 & 18.49 & 17.76 & $-0.35 \pm 0.25$ & -0.58 & $10.67 \pm 0.01$ & $11.36 \pm 0.06$ & $11.68 \pm 0.03$ & $1.01 \pm 0.04$ \\
\hline 23 & $214500.25+011157.5$ & 0.204 & 20.00 & 19.40 & $-0.63 \pm 0.14$ & -0.98 & $10.45 \pm 0.01$ & $10.67 \pm 0.13$ & $10.95 \pm 0.10$ & $0.50 \pm 0.10$ \\
\hline 24 & 231812.99-004126.1 & 0.252 & 19.23 & 18.87 & $-1.18 \pm 0.04$ & -1.51 & $10.89 \pm 0.00$ & $11.17 \pm 0.09$ & $11.53 \pm 0.06$ & $0.64 \pm 0.06$ \\
\hline 25 & $232539.22+004507.2$ & 0.277 & 20.47 & 20.25 & $-1.49 \pm 0.12$ & -1.77 & $10.44 \pm 0.01$ & $10.31 \pm 0.48$ & $10.69 \pm 0.34$ & $0.25 \pm 0.34$ \\
\hline 26 & $235347.68+005402.0$ & 0.223 & 20.07 & 19.92 & $-1.66 \pm 0.10$ & -1.83 & $10.38 \pm 0.01$ & $10.62 \pm 0.18$ & $10.91 \pm 0.12$ & $0.53 \pm 0.12$ \\
\hline
\end{tabular}

Notes.

${ }^{a}$ ID refers to the labels shown in Figure 1.

${ }^{\mathrm{b}}$ Far-IR luminosity integrated over the range $40-120 \mu \mathrm{m}$.

c IR luminosity integrated over the range 3-1000 $\mu \mathrm{m}$.

$\dagger$ Indicates objects referred to as Dominant Compact Objects in O09.

of $>2^{\prime}$. We find $A_{\mathrm{FUV}}=4.54+2.07 \beta \pm 0.4\left(\mathrm{IRX}_{\mathrm{M} 99, \text { inner }}\right.$, dotted line), and $A_{\mathrm{FUV}}=3.85+1.96 \beta \pm 0.4\left(\mathrm{IRX}_{\mathrm{M} 99, \text { total }}\right.$, dashed line). Our redetermination of IRX $\mathrm{X}_{\mathrm{M} 99 \text {,inner is very similar to the }}$ original M99 relation (IRX $\mathrm{M}_{\mathrm{M} 9,0}$, thin solid line). However, using the new larger aperture we find $\sim 2 \times$ more flux in the FUV for nearly all sources, and hence a lower IRX. The smaller IUE aperture used by M99 may thus have missed about half the light. On the other hand, in both cases we use a singleintegrated IR luminosity of unknown spatial distribution. For a subset of 12 galaxies from M99, we were able to compare the IRAS measurements with more recent determinations of $L_{\mathrm{IR}}$ based on Spitzer photometry encompassing the entire source (Engelbracht et al. 2008). The results are in good agreement. If a significant fraction of the IR is due to heating by stars further out, using $\mathrm{IRX}_{\mathrm{M} 99,0}$ rather than $\mathrm{IRX}_{\mathrm{M} 99, \text { total }}$ would lead to an overestimate of the total attenuation. Alternatively, IRX $\mathrm{M}_{\mathrm{M} 9 \text {,total }}$ could be affected by the (redder) UV light from somewhat older stellar populations on the outskirts of the galaxies. In this case, the interpretation of IRX $\mathrm{M}_{\mathrm{M} 9 \text {,total }}$ would be less straightforward as it requires knowledge of the star formation history (see Kong et al. 2004).

How do LBAs compare to these IRX relations? A fit to LBAs gives $A_{\mathrm{FUV}}=4.01+1.81 \beta$ (implied FUV attenuations of $0-3 \mathrm{mag}$ ). The dispersion found in IRX $\mathrm{X}_{\mathrm{LBA}}$ is $\sim 0.6$. As shown in Figure 1, the LBAs are thus in much better agreement, on average, with M99 (inner) than with M99 (total), but we note that this only applies to the range of $-2.5 \lesssim \beta \lesssim-1$ probed by these samples. We conclude that the dust properties of LBAs are most similar to those found only in the inner starburst cores of the M99 galaxies.

\subsection{The Attenuation of Starbursts at Low and High Redshifts}

With good estimates of $L_{\mathrm{IR}}$ and $L_{\mathrm{FUV}}$ and thus the bolometric luminosity $\left(L_{\mathrm{bol}} \equiv L_{\mathrm{FUV}}+L_{\mathrm{IR}}\right)$, we can compare LBAs to other local and high-redshift SFGs. This is shown in Figure 2. In the left panel we show $L_{\mathrm{bol}}$ versus the attenuation $\left(L_{\mathrm{IR}} / L_{\mathrm{FUV}}\right)$ for LBAs, ordinary SFGs, M99 starbursts, and luminous IR galaxies (LIRGs) all at low redshift, and "BM/BX" LBGs at $z \sim 2$ from R10. Typical SFGs in the local universe lie along a broad sequence in which the attenuation roughly follows $L_{\text {bol }}$ (indicated by the dotted line). LBAs and LBGs follow a similar relation, but one that is offset by $1-2$ dex toward lower attenuations at the same $L_{\mathrm{bol}}$ (the solid line shows the best-fit relation from R10). Conversely, at fixed $L_{\mathrm{bol}}$ LBAs and LBGs reach much higher FUV luminosities than typical local SFGs (i.e., $L_{\mathrm{FUV}} \gtrsim 0.3 L_{z=3}^{*}$, the right panel of Figure 2).

Because LBAs appear so similar compared to $z \sim 2$ LBGs in terms of their $L_{\mathrm{bol}}$ and attenuation (Figure 2), it is interesting to see whether the IRX relation at low and high redshifts is similar as well. In Figure 3, we show a compilation of low- and highredshift data for which accurate measurements are available. We first compare LBAs with the sample of $z<0.2$ SFGs detected with GALEX and Herschel from Buat et al. (2010), and with LIRGs from Howell et al. (2010), shown on left. Aperture effects either in the UV or the IR are most likely much less of a problem compared to the M99 sample, due to the higher average redshifts 


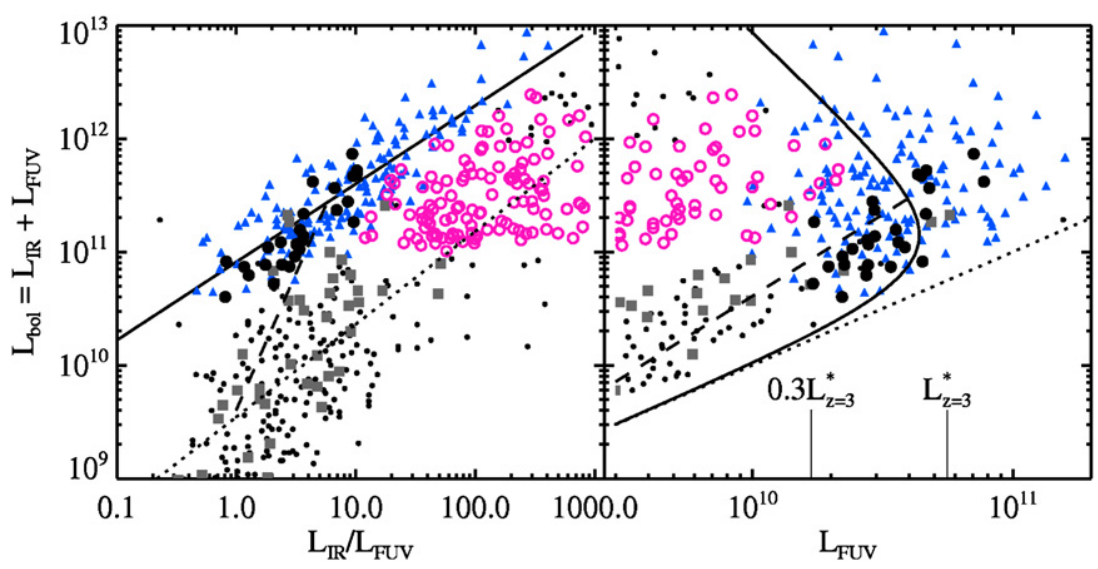

Figure 2. $L_{\mathrm{bol}}$ vs. the attenuation $\left(L_{\mathrm{IR}} / L_{\mathrm{FUV}}\right.$, left panel) and $L_{\mathrm{FUV}}$ (right panel) for LBAs (large circles), $z \sim 2$ LBGs (blue triangles, from R10), M99 (gray squares, with $R<R_{\max }$ ), LIRGs (open magenta circles; Howell et al. 2010), and ordinary SFGs (small circles; see Huang et al. 2009). The local samples (M99, SFGs, and LIRGs) lie along a broad relation between $L_{\mathrm{bol}}$ and attenuation (the result of a simple linear fit to guide the eye is indicated by the dotted line). The most extreme UV-selected samples at low (LBAs) and high redshifts (LBGs) also lie along such a relation, but one that is offset by 1-2 dex toward lower attenuations with respect to the former. The mean relation at $z \sim 2$ from R10 is indicated (solid: BM/BX sample; dashed: extrapolation to intrinsically faint objects). The fraction of $L_{\mathrm{bol}}$ emitted in the FUV is much higher for LBGs and LBAs compared to typical local SFGs (right panel).

(A color version of this figure is available in the online journal.)

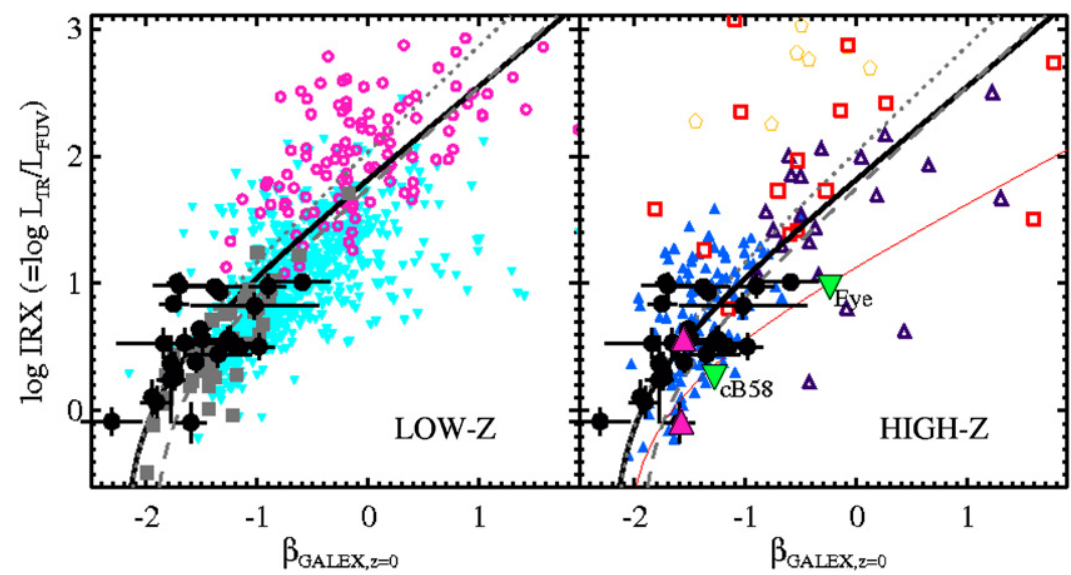

Figure 3. IRX $-\beta$ at low (left) and high redshifts (right). LBAs are indicated in both panels (black points). Left panel: M99 starbursts with $R<R_{\max }$ (gray squares), SFGs (cyan upside-down triangles; Buat et al. 2010), and (U)LIRGs (open magenta circles; Howell et al. 2010. Right panel: LBGs (filled blue triangles), BzK galaxies (open purple triangles), distant red galaxies (open red squares), and SMGs (open orange pentagons) at $z \sim 2-3$ (Reddy et al. 2006; R10), lensed LBGs at $z \sim 3$ (large green triangles; Siana et al. (2008, 2009), and statistical detections of LBGs at $z \sim 3$ detected at $1.1 \mathrm{~mm}$ (lower magenta triangle; Magdis et al. 2010a) or at 160 $\mu \mathrm{m}$ with Herschel (upper magenta triangle; Magdis et al. 2010b). Curves show IRX ${ }_{M 99 \text {,inner (dotted), IRX }}$ 99, total (dashed), and IRX $\mathrm{LBA}_{\mathrm{LB}}$ (thick black) from Figure 1 and Section 3.1. The IRX relation assuming an SMC extinction curve is indicated by the thin red line.

(A color version of this figure is available in the online journal.)

of the two samples compared to that of M99. Therefore, we will use IRX $\mathrm{X}_{\mathrm{M} 9 \text {,total }}$ as our reference (dashed line). LIRGs tend to lie above this relation, while the galaxies from Buat et al. (2010) lie below it (on average). For these samples, we would therefore tend to, respectively, under- and overestimate the attenuation when using IRX $\mathrm{X}_{\mathrm{M} 9 \text {, total }}$, while IRX $\mathrm{X}_{\mathrm{M} 9 \text {, inner }}$ performs somewhat better for LIRGs (see Buat et al. 2010; Howell et al. 2010, for detailed discussion).

In the right panel of Figure 3 we present an overview of the situation at $z>2$, showing LBGs, $B z K$, and submillimeter galaxies (SMGs) at $z=2-3$ (Reddy et al. 2006; R10), lensed LBGs ("cB58" and the cosmic "Eye"; Siana et al. 2008, 2009), and stacked LBGs at $z \sim 3$ (Magdis et al. 2010a, 2010b). LBAs and $z \sim 2$ LBGs occupy a very similar region in this IRX- $\beta$ diagram, confirming the similarity between the two samples. We should note that the IRX estimate of R10 is not based on a direct measurement of the IR emission (it is based on a combination of $L_{\mathrm{FUV}}, L_{H \alpha}$, and $L_{8}$ ), but statistical detections in the X-rays appear consistent with the extrapolated estimates of $L_{\mathrm{IR}}$ (Seibert et al. 2002; R10). We can also compare our results with the statistical detection of $z \sim 3$ LBGs in stacks at $100 \mu \mathrm{m}$ and $160 \mu \mathrm{m}$ from recent Herschel observations. This result is indicated by the upper magenta triangle, which falls right in the middle of the distribution of LBAs and $z \sim 2$ LBGs. This suggests that even for the most IR-luminous LBGs (median $L_{\text {IR }}$ of $1.6 \times 10^{12} L_{\odot}$, i.e., ULIRGs) the attenuation is exactly as expected based on the locally determined IRX (inner) relation.

Direct measurements of the mid- or far-IR emission from LBGs are available in only a handful of cases. In Figure 3, we indicate the results found for two bright, lensed LBGs at $z \sim 3$ from Siana et al. $(2008,2009)$. These objects have direct detections at (observed) 24 and $70 \mu \mathrm{m}$ (as well as $850 \mu \mathrm{m}$ and $1.2 \mathrm{~mm}$ in the case of cB58), allowing a good estimate of $L_{\mathrm{IR}}$. It has been noted that both objects appear to lie substantially below $\mathrm{IRX}_{\mathrm{M} 99,0}$, perhaps requiring a modified extinction curve 


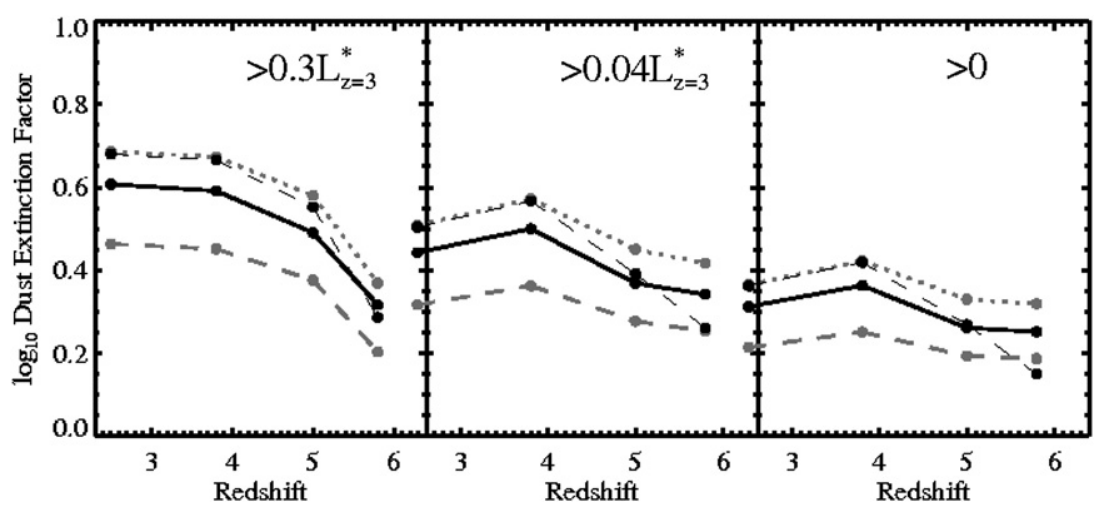

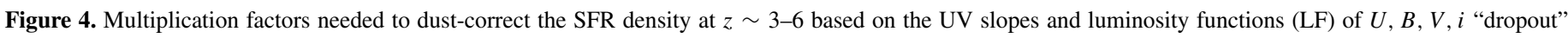

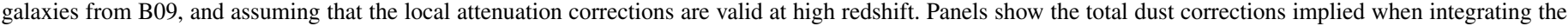

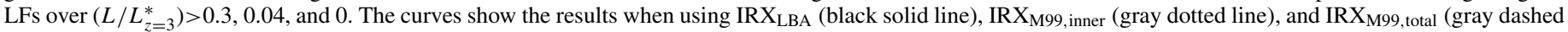
line) from Section 3.1. The thin dashed curve shows the results of B09 using the original M99 relation (IRX $\left.\mathrm{M}_{99,0}\right)$.

or an extremely young stellar population (see Baker et al. 2001; Siana et al. 2009; and the discussion on "young galaxies" by R10). An extinction curve more similar to that of the Small Magellanic Cloud (SMC), i.e., extinction that occurs in a more uniform rather than a patchy foreground dust screen would require less emission by dust for the same stellar population age, and hence lower IRX (see the red curve in Figure 3). cB58 lies on the lower envelope formed by LBAs and $z \sim 2$ galaxies, and the observed scatter is larger than the difference expected between the standard Calzetti-type and SMC-type extinction curves for a small $\beta$. The cosmic Eye, however, has a much larger slope $(\beta \approx-0.3)$ and lies well below the starburst IRX relations. Previous studies have shown that offsets from IRX are commonly due to more complicated star formation histories (e.g., Kong et al. 2004). This is a likely explanation, at least for the local SFGs (left panel), BzK galaxies (right panel; see also Nordon et al. 2010), and LBAs (see objects marked "DCOs" in Figure 1 and O09) having relatively low IRX ratios. For the cosmic Eye, however, a modified extinction curve may indeed be required in order to simultaneously explain its relatively simple stellar population and its low IRX ratio (Siana et al. 2009).

\section{DISCUSSION AND CONCLUSIONS}

The first goal of this Letter was to investigate whether LBAs are consistent with the M99 relation, and we have shown that this is indeed the case. This is an important result: because LBAs are much better analogs to LBGs compared to previous local samples, including M99, the applicability of the local dustcorrection at high redshift now appears to be on much more solid ground. Confirmation of this result is given by the fact that LBAs are also very similar to typical LBGs in terms of their dust properties (Figures 2 and 3), although some caution must be taken as true $L_{\mathrm{IR}}$ measurements are still sparse at high redshift.

We can show the implications of these results on the determination of the SFR density at $z \sim 3-6$. B09 used large dropout samples from the Hubble Ultra Deep Field to quantify the relation between $\beta_{\mathrm{UV}}$ and $M_{\mathrm{UV}}$, and computed the total dust corrections to the SFR density by integrating the UV luminosity functions (LFs) at each redshift (Bouwens et al. 2007; Reddy \& Steidel 2009) and assuming that IRX $\mathrm{M}_{\mathrm{M} 9,0}$ is valid. The method and data that we use here are similar to that of B09 (Figure 8 and Section 5.4 of that work), but instead of using IRX $_{\mathrm{M} 99,0}$ we show the results for our new IRX relations derived in Section 3.1. The results are shown in Figure 4, which gives the total correction factor that is needed to dust-correct a measurement of the UV luminosity (or SFR) density at $z \sim 3-6$. Not surprisingly, $\operatorname{IRX}_{\mathrm{LBA}}$ (thick black lines) and $\mathrm{IRX}_{\mathrm{M} 99 \text {,inner }}$ (dotted lines) give quite similar results to the relation used by B09 (thin dashed lines). Note that if we assume that IRX $_{\mathrm{M} 99 \text {,total }}$ measured within the larger aperture provides a better estimate of the total attenuation, the total dust-correction would have to be lowered by $\sim 0.2 \mathrm{dex}$ at all redshifts and for all luminosities (gray dashed lines). We conclude that current determinations of the cosmic SFR density based on dust-corrected UV data from LBG surveys appear to be on solid ground, but, as noted by B09, this is mainly due to the large contribution from very faint, blue sources at high redshift.

Direct measurements of $L_{\mathrm{IR}}$ for relatively $\operatorname{red}(\beta \gtrsim 0)$ galaxies at $z \gtrsim 2-3$ are needed to constrain the IRX relation in this regime, while the most obscured sources typically do not follow the IRX relations at all (e.g., ULIRGs at low and SMGs at high redshift). This illustrates the importance of surveys in the IR currently being performed by Herschel. In forthcoming papers we will present a more detailed analysis of the dust properties of LBAs and LBGs based on IRS spectra as well as new far-IR data from Herschel.

We thank Guinevere Kauffmann, Rychard Bouwens, Luca Cortese, and Ranga-Ram Chary for providing useful comments.

\section{REFERENCES}

Baker, A. J., Lutz, D., Genzel, R., Tacconi, L. J., \& Lehnert, M. D. 2001, A\&A, 372, L37

Basu-Zych, A. R., et al. 2007, ApJS, 173, 457

Basu-Zych, A. R., et al. 2009, ApJ, 699, L118

Boissier, S., et al. 2007, ApJS, 173, 524

Boselli, A., et al. 2010, A\&A, 518, L61

Bouwens, R. J., Illingworth, G. D., Franx, M., \& Ford, H. 2007, ApJ, 670, 928

Bouwens, R. J., et al. 2009, ApJ, 705, 936 (B09)

Buat, V., Boselli, A., Gavazzi, G., \& Bonfanti, C. 2002, A\&A, 383, 801

Buat, V., et al. 2010, MNRAS, 409, 1

Burgarella, D., Buat, V., \& Iglesias-Páramo, J. 2005, MNRAS, 360, 1413

Calzetti, D., Armus, L., Bohlin, R. C., Kinney, A. L., Koornneef, J., \& StorchiBergmann, T. 2000, ApJ, 533, 682

Carilli, C. L., et al. 2008, ApJ, 689, 883

Charlot, S., \& Fall, S. M. 2000, ApJ, 539, 718

Chary, R., \& Elbaz, D. 2001, ApJ, 556, 562

Cortese, L., Boselli, A., Franzetti, P., Decarli, R., Gavazzi, G., Boissier, S., \& Buat, V. 2008, MNRAS, 386, 1157

Engelbracht, C. W., Rieke, G. H., Gordon, K. D., Smith, J.-D. T., Werner, M. W., Moustakas, J., Willmer, C. N. A., \& Vanzi, L. 2008, ApJ, 678, 804 
Gonçalves, T. S., et al. 2010, ApJ, 724, 1373

Gordon, K. D., Clayton, G. C., Witt, A. N., \& Misselt, K. A. 2000, ApJ, 533, 236

Heckman, T. M., et al. 2005, ApJ, 619, L35

Ho, I., Wang, W.-H., Morrison, G. E., \& Miller, N. A. 2010, ApJ, 722, 1051

Hoopes, C., et al. 2007, ApJS, 173, 441

Howell, J. H., et al. 2010, ApJ, 715, 572

Huang, J.-S., et al. 2009, ApJ, 700, 183

Inoue, A. K., Buat, V., Burgarella, D., Panuzzo, P., Takeuchi, T. T., \& IglesiasPáramo, J. 2006, MNRAS, 370, 380

Johnson, B. D., et al. 2007, ApJS, 173, 392

Kong, X., Charlot, S., Brinchmann, J., \& Fall, S. M. 2004, MNRAS, 349, 769

Kurczynski, P., et al. 2010, ApJ, submitted (arXiv:1010.0290)

Leitherer, C., \& Heckman, T. M. 1995, ApJS, 96, 9

Magdis, G. E., Elbaz, D., Daddi, E., Morrison, G. E., Dickinson, M., Rigopoulou, D., Gobat, R., \& Hwang, H. S. 2010a, ApJ, 714, 1740

Magdis, G. E., et al. 2010b, ApJ, 720, L185

Meurer, G. R., Heckman, T. M., \& Calzetti, D. 1999, ApJ, 521, 64 (M99)

Nordon, R., et al. 2010, A\&A, 518, L24
Overzier, R. A., Heckman, T. M., Schiminovich, D., Basu-Zych, A., Gonçalves, T., Martin, D. C., \& Rich, R. M. 2010, ApJ, 710, 979

Overzier, R. A., et al. 2008, ApJ, 677, 37

Overzier, R. A., et al. 2009, ApJ, 706, 203 (O09)

Panuzzo, P., Granato, G. L., Buat, V., Inoue, A. K., Silva, L., Iglesias-Páramo, J., \& Bressan, A. 2007, MNRAS, 375, 640

Pope, A., et al. 2006, MNRAS, 370, 1185

Reddy, N. A., Erb, D. K., Pettini, M., Steidel, C. C., \& Shapley, A. E. 2010, ApJ, 712, 1070 (R10)

Reddy, N. A., \& Steidel, C. C. 2009, ApJ, 692, 778

Reddy, N. A., et al. 2006, ApJ, 644, 792

Rigopoulou, D., et al. 2010, MNRAS, 409, L7

Salim, S., et al. 2007, ApJS, 173, 267

Sanders, D. B., \& Mirabel, I. F. 1996, ARA\&A, 34, 749

Seibert, M., Heckman, T. M., \& Meurer, G. R. 2002, AJ, 124, 46

Seibert, M., et al. 2005, ApJ, 619, L55

Siana, B., Teplitz, H. I., Chary, R.-R., Colbert, J., \& Frayer, D. T. 2008, ApJ, 689,59

Siana, B., et al. 2009, ApJ, 698, 1273

Siebenmorgen, R., \& Krügel, E. 2007, A\&A, 461, 445

Treyer, M., et al. 2007, ApJS, 173, 256

Wang, J., et al. 2010, MNRAS, in press (arXiv:1011.0829) 